\title{
An Electronic Public Health Information System for Sri Lanka: a proposal to enhance current practice
}

\author{
Dr. G. R. M. P. Dharmawardhana MBBS, MSc \\ Medical Officer (Health Informatics), Ministry of Health, Colombo, Sri Lanka \\ E-Mail Address: manjulapra@gmail.com \\ Sri Lanka Journal of Bio-Medical Informatics 2012;3(4):132-143 \\ doi: http://dx.doi.org/10.4038/sljbmi.v3i4.5430
}

\begin{abstract}
Public health depends on timely collected, complete and accurate data. To achieve the public health goal of "collective action for sustained population-wide health improvement", the ability to measure and monitor health indices of communities and populations is very important. The Sri Lankan public health system has been exemplary among developing countries and the system is well established. Yet the growing population, increased awareness and new health needs of the community have placed a great burden on the current public health system. Reengineering of the current information system with incorporation of an Electronic Information System can be a solution for the difficulties faced by public the health staff.

In this paper, I propose to present a conceptual framework and design for an Electronic Public Health Information System. The design was made by studying the current paper based system and other practical aspects of the system. Information was gathered from field staff, middle level managers and central administrative staff. The proposed system is designed to represent all aspects of the Sri Lankan Public Health System with the ability for integration with other systems.
\end{abstract}

Keywords - Public Health Informatics; Public Health System

\section{Introduction}

"Know your area, know your people" were the words of Dr. Chellappah, the pioneer of Public Health in Sri Lanka and the Senior Medical Officer in the first health unit in Sri Lanka. Those words emanate the power of information and that information is fundamental in public health. Public health depends on timely collected, complete and accurate data. To achieve the public health goal of "collective action for sustained population-wide health improvement"(1), the ability to measure and monitor health indices of communities and populations is paramount. As such the fields of Epidemiology, Demography and Biostatistics are the key disciplines of public health ${ }^{(2)}$. The classic example of an epidemiological investigation which directly led to development of public health is the work of John Snow regarding the Cholera epidemic in Soho, England. The success of which could be attributed to careful recording of all the deaths, causes of death and the street addresses of victims ${ }^{(3)}$. This emphasises the importance of having high quality information to make effective decisions in public health.

The features of a health information system should be to integrate efforts to collect, process, report and use health information and knowledge to influence decision making, programme action and research ${ }^{(4)}$. The word "system" implies that such information gathering processes should connect all stakeholders in an organised manner. But in practice, health information systems in most countries lack such cohesion. This is the price for having developed in a piecemeal way, fashioned by administrative, economic, legal and donor pressures thus making 
them invariably highly complex ${ }^{(2)}$. To effectively make decisions regarding the health in a community, we should be considering many aspects concerning provision of health care ${ }^{(2)}$ :

- Health outcomes - which is described by indices such as mortality, morbidity, disease outbreaks, health status and disability

- Ancillary or supportive processes of health - health infrastructure, equipment and facilities, political and organisational factors, costs, human and financial issues

- Determining factors of health - socio-economic, environmental, behavioural and genetic factors

- Information on key stratifies - age groups, sex, ethnic groups, etc.

It is vital that this information is available in a connected and inter-dependent manner for efficient decision making in public health. Even though Electronic Information Systems may represent only one aspect from the above list, an ancillary or supportive process, it is vital for the functioning of all other processes.

The public health arm of health care was established in 1926 in Sri Lanka as a "health unit"(5). It has evolved since then with a well laid down information flow. The state sector is the main provider of preventive health services in Sri Lanka with very limited contribution from private sector and non-governmental organisations ${ }^{(6)}$. The public health workforce mainly consists of field staff, field and regional administrative staff or middle level managers and central administrative staff. This well established information flow is bi-directional from field to the central level establishments.

Figure 1 shows the basic information flow established in the current paper based system.

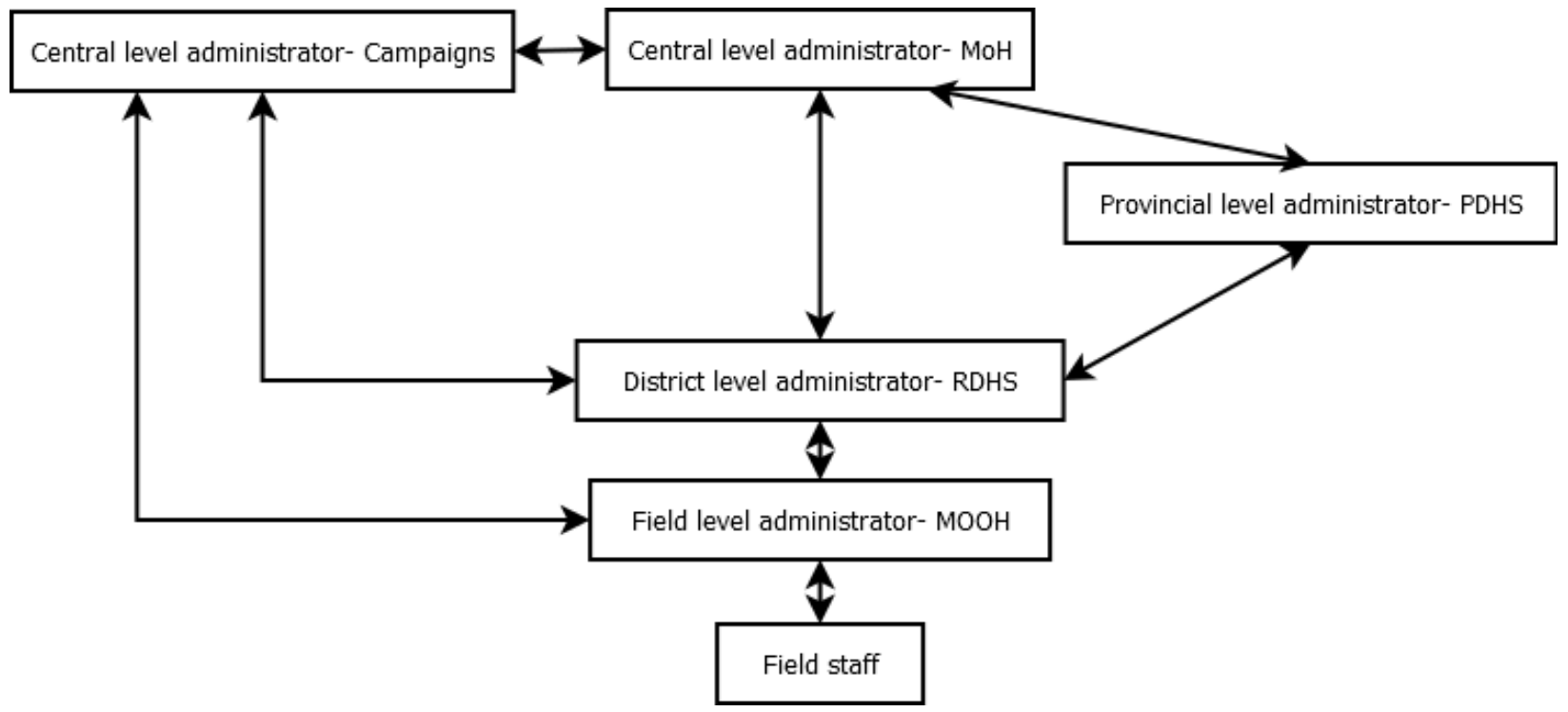

Figure 1. Current information flow 
(PDHS - Provincial Director of Health Services, RDHS - Regional Director of Health Services, MoH - Ministry of Health)

The field staff is the main point of service provision to the community and the main source of data origin. As perceived by the field staff the current information system is already burdened with many factors including their large catchment populations. Even though the middle level managers perceived having a good information system to monitor and analyse the health status as a strength of the system, the field staff consider having to collect large amounts of data as a weakness $^{(6)}$ of the current information system. Only $47 \%$ of the field staff perceived that information management is applicable to them while $100 \%$ of the administrators perceived information management is applicable to them. Field staff assessed themselves to be 3.1 (mean) competent in managing information in a scale of 0-5 while all other staff categories scaled it higher $^{(6)}$. The most recent Annual Health Statistics available are for $2007^{(7)}$. Collectively, these represent a problem within a very successful Public Health System; the current information transaction methods do not meet the current need. Data quality reflects the completeness and validity of the data recorded in the public health surveillance system ${ }^{(8)}$. Concerns have been expressed on the quality of the information collected through the system, especially with reference to health related data e.g. causes of death ${ }^{(6)}$. There is ample evidence that the current system needs to be re-engineered to cater to the growing population and the changing health care needs of the country.

\section{Objectives of a Public Health Information System (PHIS) for Sri Lanka}

The overall business goal of the proposed PHIS is to enhance the public health information gathering, recording, aggregation, communication and visualisation for quick and effective decision making, information dissemination and decision support. The objective of this system is to increase the quality of information, reduce workload, increased work satisfaction, increase availability and security of data. The business objectives for each main stakeholder were defined.

The field staff / middle level manager's business objectives for the PHIS

- $\quad$ Provide easy recording of data with data validation thus enhancing the quality of data

- $\quad$ Automated data aggregation and communication

- $\quad$ Visualisation of data pertaining to each field staff

- $\quad$ Provide easy search and data retrieval capabilities

- $\quad$ Minimise data redundancy

- $\quad$ Automated data interpretation and decision support

- $\quad$ Programme organisation and conduction

- $\quad$ Provide essential security over the collected data

- $\quad$ Provide support for research

The central level administrator business objectives for the PHIS

- $\quad$ Ability to view aggregated data or atomic data on demand

- $\quad$ Automated data interpretation and alerting

- $\quad$ Rapid data visualisation and decision support

- $\quad$ Create and manage (public health management) programmes through the system

- Quick information dissemination to the field staff 
- $\quad$ Provide support for research

- $\quad$ Publish data to the World Wide Web on demand

- $\quad$ Provide essential security

The Ministry of Health business objectives for the PHIS

- Increase the efficiency and quality of public health sector information

- $\quad$ Reduce the overall cost of information retrieval and direct it towards action

\section{The proposed information framework}

The proposed information framework is designed considering many organisational, practical, social and personal issues. Non availability of internet facilities at the field level was a practical problem at field setting. Properly instituted computing infrastructure is yet to be implemented in the health sector in Sri Lanka but basic computing facilities are available in most administrative level institutions and in some cases even at the field staff level. This was taken into consideration when designing the information structure. Databases are present at each level of data origination which is synchronised with a central database server. This would serve both as a data repository and a backup mechanism. There is a separate aggregated data flow which is routinely active. Figure 2 summarises the proposed information framework.

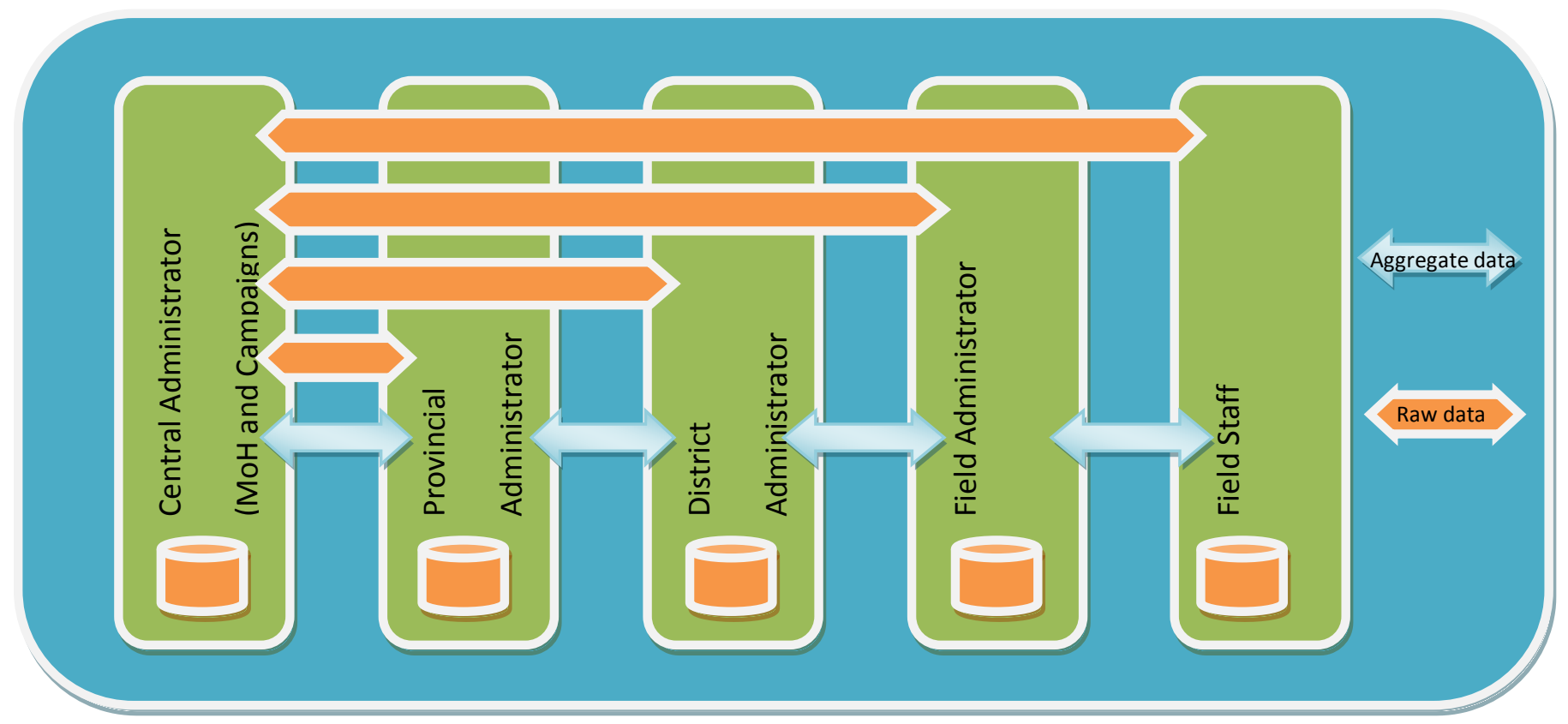

Figure 2. Proposed information flow

\section{Designing the system}

The proposed system design mirrors the information flow. There will be separately distributed systems for each level of information flow and each system is capable of functioning on its own. 
The communication between each level will be as data and information. Figure 3 depicts the context diagram of proposed system.

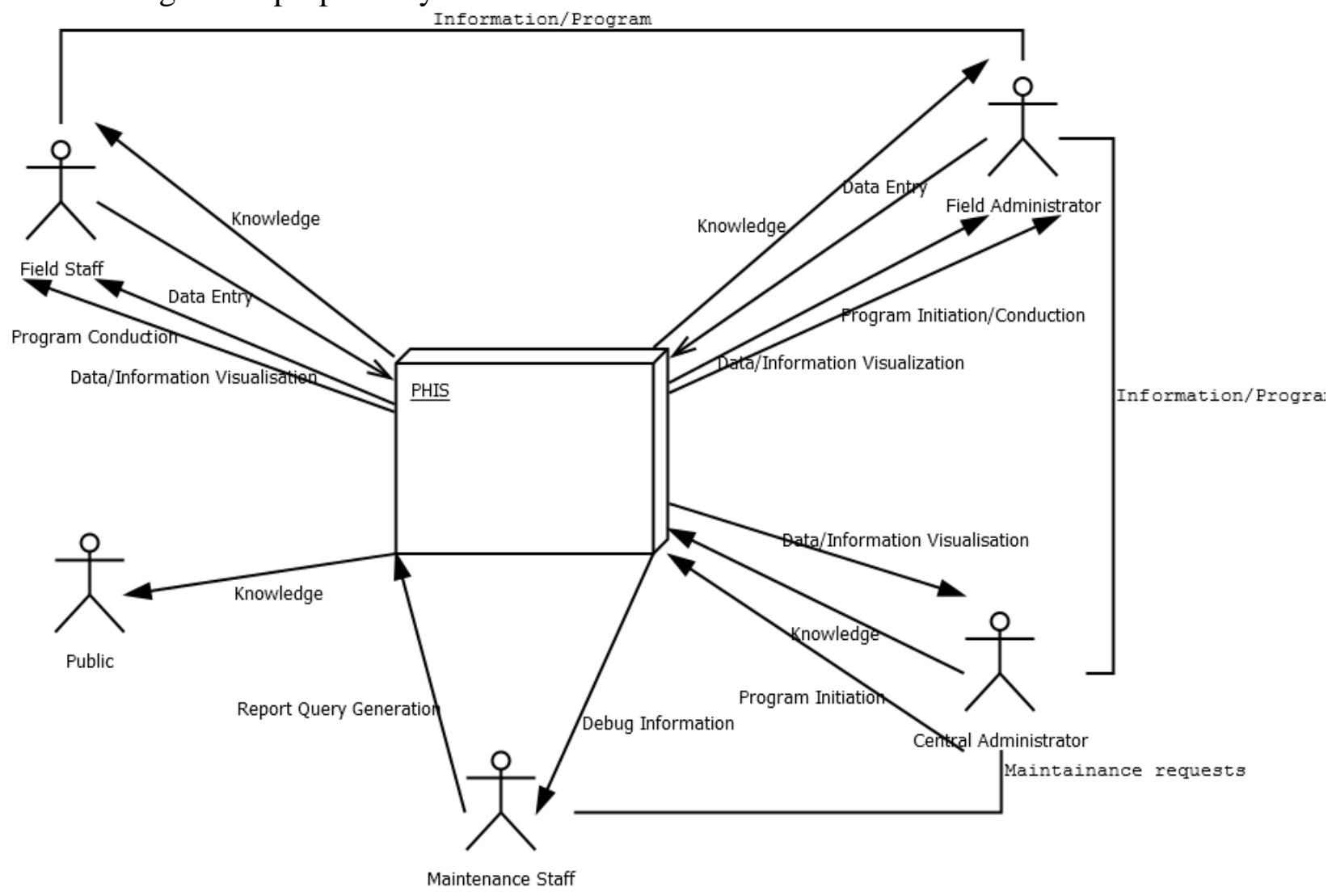

Figure 3. Context diagram of PHIS

\section{System capabilities}

Following is a comprehensive list of proposed system capabilities listed according to the user roles.

The PHIS will provide the following capabilities to field staff

- Login to the system securely

- View and / or enter data related to the range or area

- Edit data which are not yet aggregated

- Enter GIS data if available

- Generate, view and save aggregate data

- Generate cumulative reports, print them

- Generate graphs or other visual representation of data

- View and update programme information

- View published data in detail regarding range, Medical Officer of Health (MOH) area, district, province and at national level

- View help documentation of the system 
- Logout of the system

Field Administrative Officer capabilities

- Login to the system securely

- View and / or enter data

- Edit atomic data which has not being aggregated

- View atomic data entered by field staff

- Create cumulative reports, graphs and other visual representations

- Generate information (decisions / guidelines or other messages) and disseminate it to subordinate field staff

- Organise programmes and host them on the system

- Monitor progression of programmes and generate alerts, inquiries

- Update programmes set by central level

- Log out of the system

The PHIS should provide the following capabilities to central administrators

- Login to the system securely

- View all the aggregated data

- View atomic data by selecting through the required region on demand

- Generate cumulative reports, graphs and other visual representation

- Generate information (decisions / guidelines or other messages) and disseminate it to field staff / field administrators

- Generate information and publish in world wide web

- Organise programmes and host them on the system

- Monitor progression of programmes and generate alerts, inquiries

- Log out of the system

PHIS should provide following capabilities to maintenance staff

- Log in to the system securely

- Create / edit users of the system.

- Create / edit data validation rules

- Add / edit aggregation calculation rules

- Add / edit report generation logic using a logical syntax code

- Create / change backup rules

- Analyse crash reports and rectify issues

- Create / edit version mapping rules.

- Create / edit decision support engine rules

PHIS will be providing the following facilities for the general public

- View information posted to the web portal of the system

\section{Other capabilities}


- Automated database synchronisation. All the databases at each user will be mirrored in the central server. The PHIS will automatically synchronise data once the system is online. It will have the ability to monitor network status. Databases at each field level, district and provincial levels should be synchronised with the central server

- Semi automated aggregation calculation and upload PHIS will calculate aggregates and upload them to the database of each upper level hierarchy database. In a situation with no connectivity, it will have the ability to create a file according to the user's recommendation. This file may be emailed or handed over physically to the relevant officer. PHIS will also have the ability to import the file once its physical location is given

- Automated capturing of geological information PHIS will have the ability to capture information from Global Positioning System (GPS) when such data is available in the device used. It will allow manual entering of such information if such information is available at the data entry point but not integrated with the data input device

- Automated data analysis and decision support PHIS will have the ability to automatically analyse data continuously. It will identify trends, outliners and other important information from data as specified in the rules file for data interpretation and notify the specified users when they $\log$ in to the system. For very important information as specified in rules, PHIS will send an email to the specific user

- Semi automatic crash reporting when the system experiences a crash or unintended behaviour it will generate a crash report with system information, activity done before crash and other technical information. This will be saved as a file and if system is online it will submit the report to the central server following user authorisation and in a situation where there is no connectivity it will allow user to either email the file or hand it over physically to the maintenance staff

- Automatic module update PHIS will have the ability to maintain a version reference in every computer in which it is used and will update the system automatically when the former system goes online. In a case of version discrepancy where already entered data is present, it will use the assistance of a version mapping reference file to decide how it will save the older data to the newer database

\section{Solving unique field level difficulties}

The design of the system was carried out to meet all the objectives defined. Several measures were taken to solve problems unique at the field level.

To increase the opportunity of capturing data, data entry is allowed in every stage except the central level. This decision was taken to curb the problems arising due to unavailability of computer facilities. It is important to note that aggregate data generated at the lower level of the 
hierarchy becomes the atomic data at the next level. Data entry to the system was possible using this strategy at every level.

One of the major problems faced at the field is lack of internet facilities. Even though the situation is rapidly changing ${ }^{(9)}$, it will be a problem for many years to come. This proposed system boasts an online / offline system where the system will be capturing information even when it is offline. It will synchronise the database and upload the aggregate information as soon as a communication channel becomes available. In a situation where there is no internet connectivity for longer durations; system will allow the user to manually submit information by exporting and importing to removable media. One major reason for system abandonment at the field level is hardware failure and loss of data. PHIS will have all databases of each user synchronised to a central database and the central database will be having backup plans. There will be a two way synchronisation ensuring no loss of data within the system.

\section{The proposed software architecture}

PHIS will be designed using a modular architecture, as modular approach in software design has shown clear benefits. Some scholars perceive modules of a software as "transformers of data" while others think that "a component is a unit of software that performs some function at runtime" ${ }^{(10)}$. Both views were considered when breaking the system to modules. Every major task according to the system capabilities was broken to manageable modules. Following rules were applied when breaking the tasks into modules.

1. The module should handle one specific business process

2. A module should be small so that it could be downloaded over the internet

3. If a reference file is used, one module should use only one file

Figure 4 illustrates the module integration and interaction. 


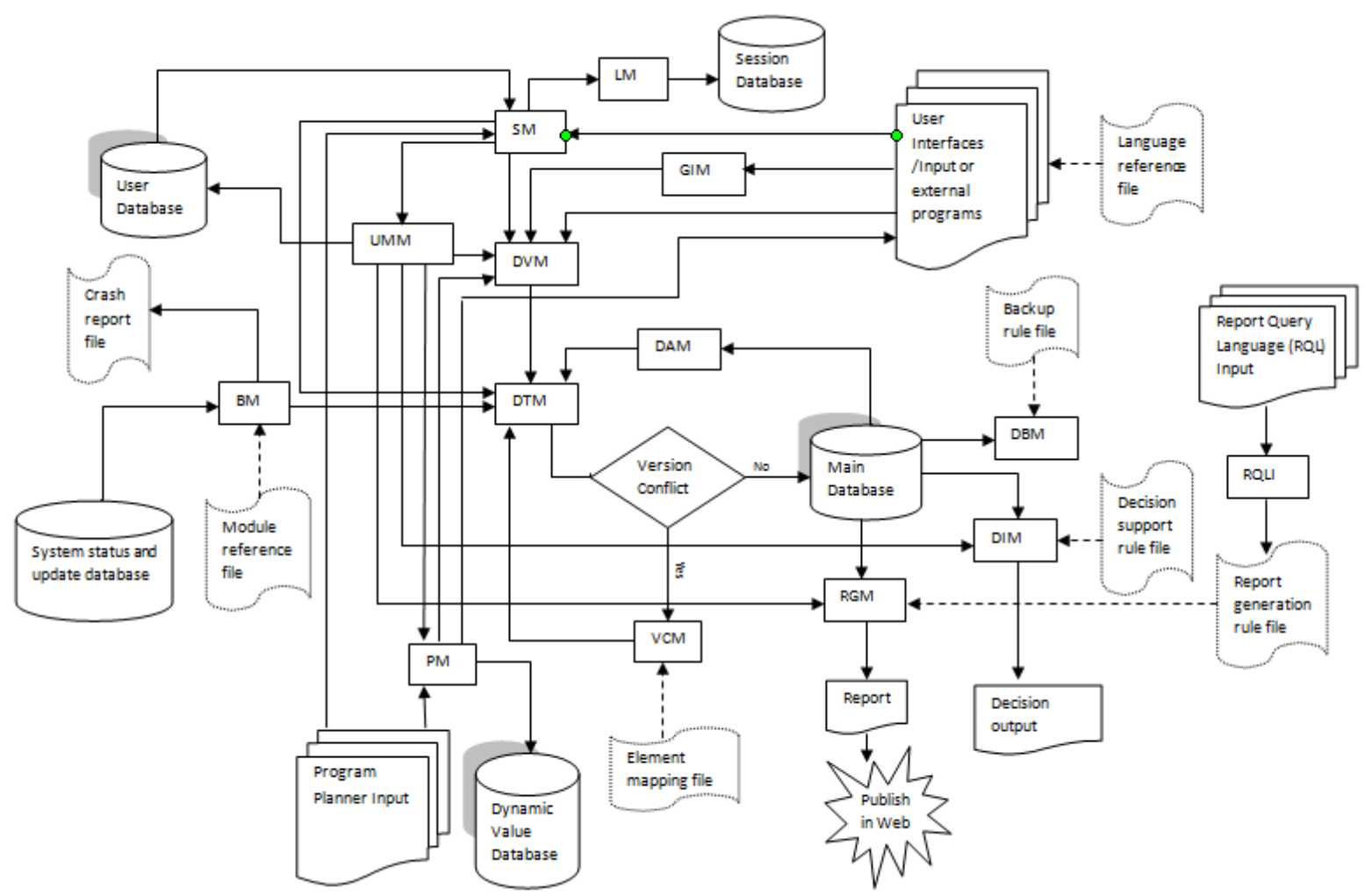

Figure 4. Module integration and interaction

Following is short description of the modules

1. Base Module (BM)

a. Check system integrity and start the programme

b. Run and / or download and install other modules

c. System wide notification of online status

d. Check for updates download and install

2. Security Module (SM)

a. Authentication of users

b. Database security

3. User management module (UMM)

a. Data validation rule generation for users

b. Add / edit users

4. Data validation module (DVM)

a. Data input validation module

5. Data accumulation module (DAM)

a. Calculate aggregate data

6. Data transfer / synchronisation module (DTM)

a. Synchronise data/aggregated data between databases

7. Database backup module (DBM)

a. Perform database backup at the central level

8. Report generation module (RGM)

a. Contains the algorithms for report generation

9. GIS integration and mapping module (GIM) 
a. Handle GIS data and integration with other data

10. Data interpretation module (DIM)

a. Interpret data

11. Version Compatibility Module (VCM)

a. Resolve compatibility issues with module versions

12. Logging module (LM)

a. Log session information

13. Report Query Language (RQL) Interpreter (RQLI)

a. Interprets the report query language and amends the Report Generation Rule file 14. Programme manager

a. Update activities, deadlines and targets regarding a planned programme

b. Provide alerts to the user regarding activities, deadlines and targets

c. Provide dynamic interface information for additional data elements

\section{Connecting with outside systems}

The design of the system was carried out considering all the aspects of public health considering information which are not yet available in the information flow infrastructure, such as primary care, over the counter drugs, pattern of service seeking and access of lab facilities. The system will be following the HL7 messaging framework and this will allow outside systems to communicate with the PHIS as and when required with proper authentication mechanisms.

In Sri Lanka, the public health services are mainly focused on the provision of preventive and promotive health services and do not include first contact medical care ${ }^{(6)}$. This is one of the major drawbacks of the current public health information system. It is important to capture this hospital information at least when getting recorded in electronic format ${ }^{(11)}$ and integrating this information to PHIS can be done once other systems are developed on data communication standards or interfaces are developed. This will add a new dimension to the Sri Lankan public health system. Another system for recording first contact medical care at hospital setting, is also being piloted $^{(12)}$ and statistical data derived from such systems may also be integrated in the same manner.

\section{Discussion}

A good public health information system should serve all stakeholders of the public health in a country. Public health has always benefitted from informatics solutions which aid not only surveillance but also emergency response, environmental health and administration ${ }^{(13)}$. Out of many, the key features of a good surveillance system are the simplicity, flexibility, data quality, acceptability, timeliness and stability ${ }^{(8)}$, and should also be key features of a comprehensive information system. PHIS arrive at these parameters effectively. The simplicity is achieved by keeping data collection a high priority with increased opportunity for data entry. The system is very flexible allowing any changes, from data element change to reporting framework, even a change in the information flow can be achieved with few measures, importantly without the involvement of a programmer. Measures taken for improved data quality comprise of data validation procedures and data synchronisation. Acceptability is a key facture influencing long 
term sustainability of a system. PHIS tries to increase the acceptance by easy and non-redundant data entry, increased reliability, feedback on individual and aggregated data, increased security over data, acknowledgement of the contribution of the staff and dissemination of aggregated data. Timeliness of the data can be achieved by reminding the user on timely entry of data with automated aggregation and dissemination. System stability will not compromise with error reporting methods and two way synchronisation of data.

\section{Conclusion}

The vision of using Health Informatics for public health is to improve the high-value data, information, and knowledge to be exchanged in a secure and timely manner. WHO has stated that "Reporting of notifiable diseases making use of modern communication technology and reporting of statistics from district to national levels in a web-based method" as one of the core indicators of country health performance ${ }^{(14)}$. As a country that is rapidly emerging as a model developing country, Sri Lanka needs to be in the forefront delivering quality health care to its citizens as it always has been. It is not because countries are poor that they cannot afford good health information systems; it is because they are poor that they cannot afford to be without it ${ }^{(2)}$.

\section{References}

1. Beaglehole R, Bonita R, Horton R, Adams O, McKee M. Public health in the new era: improving health through collective action. Lancet. 2004; 363(9426): 2084-6. doi: http://dx.doi.org/10.1016/S0140-6736(04)16461-1

2. AbouZahr C, Boerma T. Health information systems: the foundations of public health. Bulletin of the World Health Organization 2005; 83(8): 578-83.

3. Snow J, Frost WH, Richardson SBW. Snow on cholera. Humphrey Milford, Oxford University Press 1936.

4. Lippeveld T. Routine health information systems: the glue of a unified health system. Keynote address at the Workshop on Issues and Innovation in Routine Health Information in Developing Countries, Potomac 2000; 114-6.

4. Soma H. Sri Lanka's approach to Primary Health Care: a success story in South Asia. Galle Medical Journal 2011; 16(2): 24-30 doi: http://dx.doi.org/10.4038/gmj.v16i2.3749

6. World Health Organization, WHO Country Office for Sri Lanka, Health System Research Unit. Report of rapid assessment of essential public health functions Sri Lanka. World Health Organization; 2006.

Available from: http://whosrilanka.healthrepository.org/handle/123456789/ 243

7. Ministry of Health. Annual Health Bulletin. Available from: http://www.health.gov.lk/ AnnualHealthBulletin.htm 
8. German RR, Lee L, Horan J, Milstein R, Pertowski C, et al. Updated guidelines for evaluating public health surveillance systems. MMWR 2001; 50: 1-35.

9. Telecommunications Regulatory Commission of Sri Lanka. Statistical Overview. Telecommunications Regulatory Commission of Sri Lanka; 2012. Available from: http://www.trc.gov.lk/information/statistics.html

10. Fielding RT. Architectural styles and the design of network-based software architectures. University of California. 2000.

11. Kariyawasam NC, Weerasekera VS, Dayaratne MKDRB, Hewapathirana R, Karunapema RPP, et al. eIMMR: the future of health statistics in Sri Lanka. Sri Lanka Journal of BioMedical Informatics. e-Health Sri Lanka 2010; 1(suppl 1):S14 doi: http://dx.doi.org/10.4038/sljbmi.v1i0.3549

12. Information and Communication Technology Agency of Sri Lanka. Automation of patient information in Government hospitals [Internet]. Available from: http://www.icta.lk/en/icta/90-general/1025-automation-of-patient-information-ingovernment-hospitals.html

13. Thomas G, Savel TG, Foldy S, others. The Role of Public Health Informatics in Enhancing Public Health Surveillance. CDC's Vision for Public Health Surveillance in the 21st Century. 2012; 61(3): 20-24.

14. World Health Organization. Health information systems. World Health Organization; 2008. Available from: http://www.who.int/healthinfo/statistics/toolkit_hss/EN_PDF_Toolkit_HSS_InformationSy stems.pdf 